Case Report

\title{
Ameloblastic Carcinoma of the Mandible: a Rare Case Report
}

\author{
Mandadi dakshina murthy Srikanth ${ }^{1}$, Radhika Besta ${ }^{2}$, Kiran kumar Metta ${ }^{3}$, Nukala \\ valli Renuka ${ }^{4}$. \\ ${ }^{1,2}$ Department Of Oral Medicine and Radiology, MNR Dental College and Hospital, Fasalwadi, \\ Sangareddy,India \\ ${ }^{3}$ Department of conservative dentistry and Endodontics, MIDSR dental college and hospital, Latur, \\ Maharashtra,India \\ ${ }^{4}$ Department of periodontics, Army College of dental sciences, Secunderabad, India \\ Correspondence should be addressed to: Mandadi dakshina murthy Srikanth; \\ drsrikanthmd@rediffmail.com
}

Received date: 13 Mai 2014; Accepted date: 4 June 2014; Published date: 27 May 2015

Academic Editor: Keisuke Nakano

Copyright (C) 2015. Mandadi dakshina murthy Srikanth, Radhika Besta, Kiran kumar Metta, Nukala valli Renuka. Distributed under Creative Commons CC-BY 4.0

\begin{abstract}
Ameloblastic carcinoma is characterized by rapid, painful expansion of the jaw, unlike conventional ameloblastoma. The tumor most frequently involves the mandible. The expanding lesion causes perforation of buccal and lingual plates of the jaw and invades the surrounding soft tissue. Rapidly growing large tumor mass may cause tooth mobility. Mandibular tumor involving mental nerve leads to the paresthesia of the nerve. Maxillary tumor can produce fistula in the palate and paresthesia of infraorbital nerve. Although rare, these lesions have been known to metastasize mostly to the regional lymph nodes or lungs. Because the lesion is usually found unexpectedly after an incisional biopsy or the removal of a cyst, a guide to the differential diagnosis is usually not useful. The tumor cells resemble the cells seen in ameloblastoma, but they show cytologic atypia. Moreover, they lack the characteristic arrangement seen in ameloblastoma. Direct extensions of the tumor, lymph node involvement have been reported. A case of ameloblastic carcinoma in a 60 year old man of typical aggressiveness and extensive local destruction is reported here and its clinical, radiographical and histological features are discussed.
\end{abstract}

Keywords: Ameloblastic Carcinoma, squamous Metaplasia.

Cite this Article as: Mandadi dakshina murthy Srikanth, Radhika Besta, Kiran kumar Metta, Nukala valli Renuka (2015)," Ameloblastic Carcinoma of the Mandible: a Rare Case Report", Journal of Research and Practice in Dentistry, Vol. 2015 (2015), Article ID 672596, DOI: 10.5171/2015.672596 


\section{Introduction}

Malignant odontogenic tumors are very uncommon and ameloblastic carcinoma is a rare odontogenic carcinoma with very few such cases being reported so far ${ }^{1}$. The frequency of malignant change in ameloblastoma is difficult to establish but probably may be less than $1 \%$ among all cases of ameloblastomas ${ }^{2}$.

The importance of the discussion for ameloblastic carcinoma is important to the fact that the terminology for these lesions is somewhat controversial. The question of malignancy in ameloblastoma has been the subject of considerable discussion for many years. The instances suggest that ameloblastoma has been considered malignant on the basis of aggressive clinical course in the absence of metastasis. These lesions may follow markedly aggressive local course, but metastases do not necessarily occur $^{3}$.In the updated histologic classification of the world health organization in 2005, ameloblastic carcinoma is classified as ameloblastic carcinoma, primary type; ameloblastic carcinoma, secondary type (dedifferentiated), intraosseous; and ameloblastic carcinoma, secondary type (dedifferentiated) peripheral carcinoma. It may be represented as a cystic lesion with benign clinical features or as a large tissue mass with ulceration, significant bone resorption, and tooth mobility. Such lesions are usually detected unexpectedly after an incisional biopsy or following the removal of a cyst and hence a guide to the differential diagnosis is not usually useful ${ }^{3}$.

Ameloblastomic carcinoma is a rare malignant lesion with characteristic histologic features and behavior that dictates a more aggressive surgical approach than that of a simple ameloblastoma. The identifying features of ameloblastic carcinoma must be known and recognized by dental practitioners. The tumor cells resemble the cells seen in ameloblastoma, but they show cytologic atypia. Moreover, they lack the characteristic arrangement seen in ameloblastoma ${ }^{4}$.

\section{Case Report}

A male patient aged 60 years, came to the department of oral medicine and radiology, with a chief complaint of swelling over the right side of the face since 10 years. History revealed that he first noticed a small intra oral swelling at the labial aspect of the lower right canine region which gradually increased in size. Initially, he noticed pain at that region but subsequently but there was no pain and the swelling increased progressively in size and attained the present size. The patient also noticed development of paresthesia of the lower lip with pain over the swelling.

On examination there is a huge extra oral swelling measuring around $23 \mathrm{cms} \times 11.5 \mathrm{~cm}$ in size extending from the right side of the mandible and crossing the midline with well defined margins and hard in consistency with tenderness over the swelling and intra oral examination revealed complete obliteration of the buccal and labial vestibule on the right side with the swelling extending in to the anterior region of the floor of the mouth (Fig1 ). It has normal mucosal colour and 31-33, 41-47 teeth are missing. The intra oral swelling is hard in consistency and tenderness is present on palpation. 


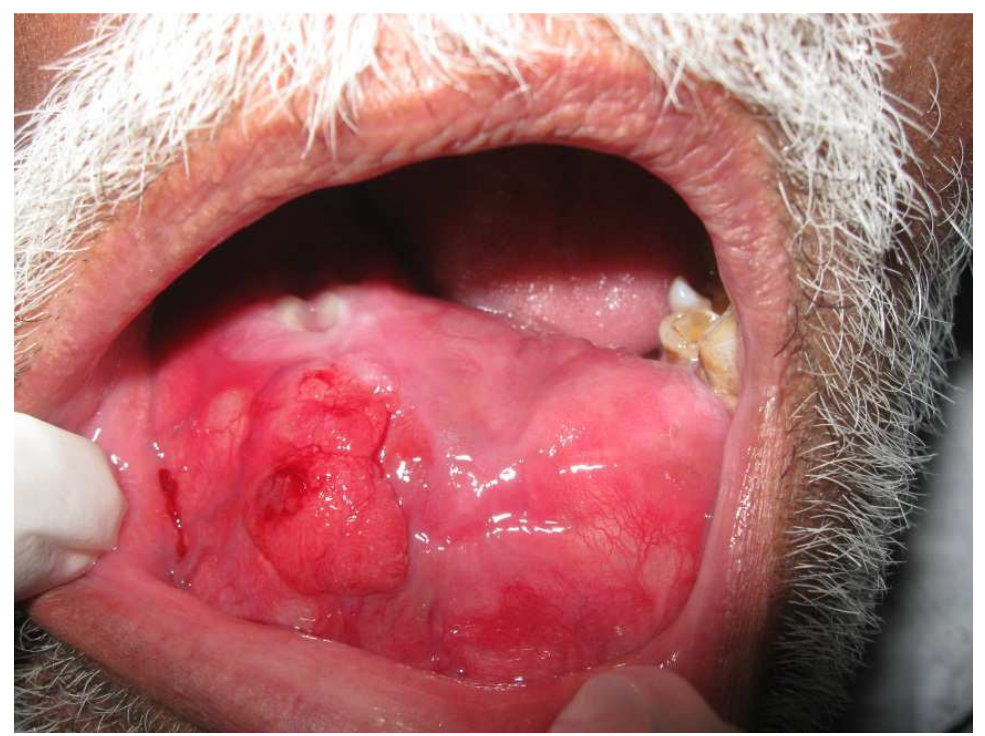

Figure 1: Intra Oral Photograpgh of Swelling with Labial, Buccal And Lingual Cortical Expansions

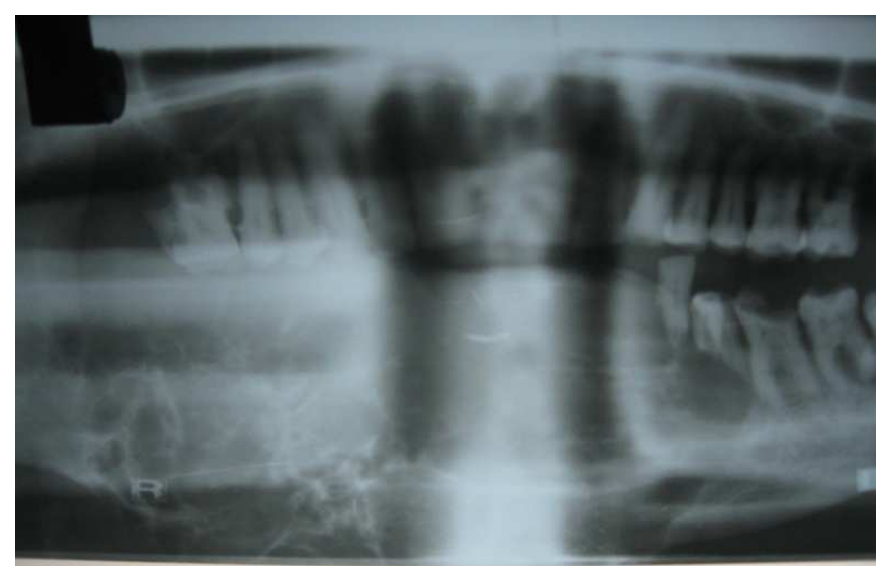

Figure 2: Opg Showing Honey Comb Lesion

CT scan showed an enlarged tumor extending from the ramus region of the lower right third molar region till the lower left second premolar region. The tumor caused severe expansion of the buccal and lingual cortical plates with multilocular appearance.
Hematoxylin and eosin sections of biopsy revealed nests and cords of odontogenic epithelium and stellate reticulum like cells. The individual cells showed nuclear and cellular pleomorphism, hyperchromatic nuclei, vesiculated nuclei with prominent nucleoli, altered nuclear cytoplasmic ratio 
and abnormal mitotic figures, which supported the diagnosis of ameloblastic carcinoma. Sections also showed follicles of odontogenic epithelium lined peripherally by tall columnar cells and central stellate reticulum like cells within a scanty connective stroma. Follicles showed varying histologic features like nuclear polymorphism, basal cell hyperplasia, squamous metaplasia with dyskeratosis, necrosis, and cystic degeneration. The surface showed parakeratinized stratified squamous epithelium of gingiva features suggestive of ameloblastic carcinoma. (Fig$3,4,5)$. Hence, a final diagnosis of ameloblastic carcinoma was arrived at. The patient was operated with surgical resection of the tumor by micro vascular reconstructive surgery with complete resection of the mandible and reconstruction of the mandible was done by fibula graft. The patient is being followed up closely since 1year.

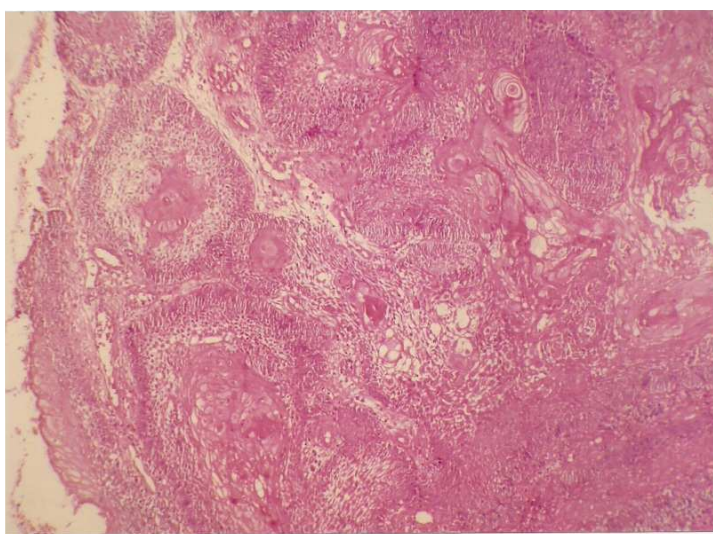

Figure 3: Histopathology Specimen Showing Epithelial Follicles with Squamous Metaplasia and Numerous Keratin Pearls.
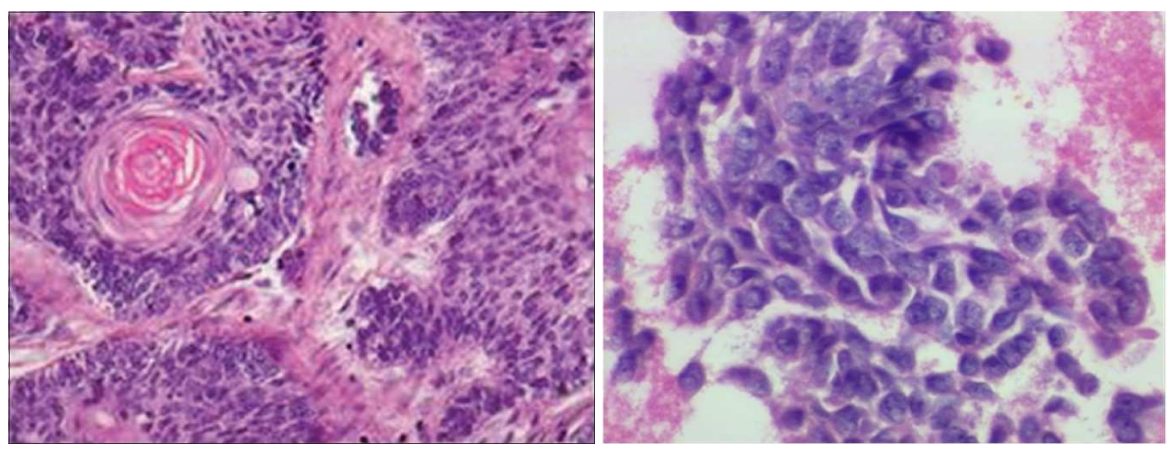

Figure 4 and 5: Magnified Images With Cells Showing Nuclear And Cellular Pleomorphism, Hyperchromatic Nuclei, Vesiculated Nuclei With Prominent Nucleoli, Altered NuclearCytoplasmic Ratio, And Abnormal Mitotic Figures. 


\section{Discussion}

The demographic data of ameloblastic carcinoma reported in the literature suggest that it is more common in males, (M: F 1.5:1) and the site of distribution is in the mandible, particularly in the posterior mandible 5 . Age range of occurrence shows large variation with an average age of 39.8 years. However, few authors stated that the sixth decade is the predominant age group. Ameloblastic carcinoma has been reported to arise either de novo, or from a preexisting odontogenic cyst, or ex ameloblastoma ${ }^{6}$.

The common clinical signs and symptoms include swelling, pain, trismus and dysphonia4. In 1983, Shafer introduced the term ameloblastic carcinoma to describe ameloblastomas in which there had been histologic malignant transformation. The characterization of carcinoma arising centrally within the mandible and the maxilla is an uncommon but complex problem. The first step in the staging process must be the exclusion of metastasis or invasion of bone by tumor from adjacent soft tissues or paranasal sinuses. The neoplasm may be derived from a number of different sources, such as those of odontogenic origin, including ameloblastoma, odontogenic cysts or epithelial odontogenic rests, as well as entrapped salivary gland epithelium or epithelium entrapped along embryonic fusion sites. Carcinomas in the jaws metastasizing from primary locations such as the lung, the breast and the gastro intestinal tract may mimic ameloblastic carcinoma and must always be ruled out clinically before that diagnosis is made.

The diagnostic criteria of an ameloblastic carcinoma that dedifferentiates from ameloblastoma are based on cytologic atypia and increased mitotic index ${ }^{6}$. The histologic changes should include, higher proliferative index emphasized by higher mitotic activity, higher proliferating cell nuclear antigen expression and higher ki67, this should be correlated with the clinical features. The four important characteristics include growth rate, the propensity for ameloblastic carcinoma to perforate the cortex, pain-as $1 / 3$ rds of patients with ameloblastic carcinoma experience pain or discomfort, sensory disturbance, such as paresthesia which is rare with ameloblastoma ${ }^{7}$. Primary intra-alveolar epidermoid carcinoma must be considered in the differential diagnosis of ameloblastic carcinoma. This tumor, developing within bone, probably originates from odontogenic epithelial remnants. According to Shear, these lesions occur over a wide age range, but most occur in the sixth to seventh decade of life. Although the primary intra-alveolar carcinoma and ameloblastic carcinoma exhibit some clinical differences, their histologic features are similar enough to suggest a histogenic relation. It is possible, then, that the primary intra alveolar carcinoma may represent simply a less-differentiated, usually a nonkeratinizing form of ameloblastic carcinoma, both lesions being derived from odontogenic remnants ${ }^{8}$.

Thus, the term ameloblastic carcinoma can be applied to our case, which showed focal histologic evidence of malignant disease including cytologic atypia and mitosis with indisputable features of classic ameloblatoma. Nevertheless, it is important, in the future, these lesions can be accurately identified, differentiated from malignant ameloblastoma and followed so that their natural history and prognosis can be further defined ${ }^{8}$.

To conclude finally, it is reasonable to consider this case as it illustrates the malignant portion in the spectrum of ameloblatomas. when the diagnosis of ameloblatic carcinoma is made, an assessment of nodal metastasis and evidence of distant metastasis is required. A staged work-up consisting of neck examination is necessary. Meticulous follow up is essential because recurrence and metastasis in the lung and regional lymph nodes have been reported. Presurgical radiation therapy has 
been suggested to decrease tumor size but chemotherapy is as yet unproven. Reconstruction of the post-resection defect may proceed as one would normally expect following any head or neck carcinoma resection. Sufficient time should be allotted before reconstruction because of potential tumor recurrence ${ }^{8}$.

\section{References}

1. Rajendran and Sivapathasundharam. Shafer's textbook of oral pathology. $6^{\text {th }}$ ed.India: Elsevier publisher; 2009.(PMID:HEALTH:1)

2. Neville, Damm, Allen. Oral \&maxillofacial pathology.3rd edition. India: Saunders/Elsevier; 2009.

3. Wood and Goaz. Differential diagnosis of oral and maxillofacial region. $5^{\text {the }}$ dition.St.Louis.Mosby

1997.(PMID:3,28)
4. Ameloblastic carcinoma of the mandible resembling Odontogenic cyst in a panoramic radiograph. Allan G.Farman. OOOE 2006; 101(5): 638-642.

5. Ameloblastic carcinoma: an analysis of 14 cases. James M Hall. O0OE 2007; 103(6):799807.(PMID:HEALTH:1)

6. Ameloblastic carcinoma: Report of a new case, literature review and comparison to ameloblastoma-Sharon Akrish .Journal of oral and maxillofacial surgery 2007; 65: 777783.

7. Ameloblastic carcinoma of the jaws-a report of two cases-Stephan $\mathrm{k}$ lay. OOOE 1998; 85:78-81.

8. Ameloblatic carcinoma: Case report and literature review-Sylvie Lousie Avon,John Mc Comb,Cameron Clokie.Journal of Canadian dental assosiation2003;69(9):573-6. 